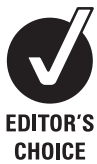

CHOICE

\title{
Making choices: why parents present to the emergency department for non-urgent care
}

\author{
A Williams, ${ }^{1}$ P O'Rourke, ${ }^{2}$ S Keogh'
}

${ }^{1}$ Nursing Research Unit, Royal Children's Hospital, Brisbane,

Queensland, Australia;

${ }^{2}$ Queensland Institute of

Medical Research, Brisbane,

Oueensland, Australia

Correspondence to:

Ms A Williams, Nursing

Research, Ground Floor, North

Tower, Royal Children's Hospital,

Herston Road, Herston 4029,

Queensland, Australia;

alison williams@health.qld.gov. $\mathrm{au}$

Accepted 8 April 2009

Published Online First

23 April 2009

\section{ABSTRACT}

Objectives: This study aims to provide a better understanding of the motivations and actions of parents of children with non-urgent injury or illness who attend the emergency department at a tertiary paediatric hospital seeking care.

Design: A prospective questionnaire-based survey of 355 parents aimed to ascertain information about parent caregiving and care-seeking behaviours prior to presentation at the emergency department with their child.

Results: A total of 355 parents were surveyed, representing $8 \%$ of the parents/carers presenting to the emergency department in a 3-month period for nonurgent (Australasian Triage System 4 and 5) care of their child. The factors identified were: parents rated their child's condition as moderate to very serious (242 (68\%)); two-thirds of parents (234 (66\%)) had sought advice prior to attending the emergency department; $54 \%$ (77) of the 137 children who attended with an injury presented promptly to emergency (ie, within $4 \mathrm{~h}$ of injury) whereas of the 216 presenting with an illness, $41 \%$ (88) presented within 2-7 days of the onset of the illness.

Conclusions: This study displayed the accuracy of "parental triage," that is, parents assess their child's health and generally engage in appropriate care-giving and care-seeking behaviours before presenting to a paediatric emergency department. Highlighted are the deficiencies in current primary care services available to families and the perception that not all cases deemed as non-urgent by the emergency department are able to be dealt with in a primary care setting.

Emergency department usage for non-urgent care has been identified as an issue worldwide. Queensland, as the fastest growing state in Australia, is no exception to this trend, with presentations to the Royal Children's Hospital Paediatric Emergency Department (PED) increased by $40 \%$ over the past 3 years (Royal Children's Hospital Department of Emergency Medicine Statisics Brisbane, personal communication, October 2008). By considering the health-seeking characteristics and behaviours of parents, it is possible that a better understanding of service delivery requirements within hospital and community settings could be gained.

\section{OBJECTIVES}

The primary aim of this study was to provide a comprehensive, systematic understanding of the motivations and actions of parents of children with non-urgent illnesses who attend a PED. This included the events that preceded presentation, including the care, advice and treatment sought and given. The specific research question that was

\section{What is already known on this topic}

- Emergency department usage for non-urgent care is a worldwide issue.

- PED presentations at the study hospital have increased by $40 \%$ over the last 3 years.

\section{What this study adds}

- There are deficiencies in primary care services

- Presentations are multifactorial and not related to socio-economic factors.

explored was: "What care-giving and care-seeking behaviours preceded parents' decisions to take their children to a PED for non-urgent care?" A secondary aim of this study was to compare the study population with that of the wider population group to gain a true interpretation of demographic features that have been said to influence attendance at a PED.

\section{DESIGN}

\section{Method and setting}

This was a quantitative study utilising a crosssectional descriptive survey design and conducted in a paediatric emergency department of a metropolitan tertiary referral paediatric hospital in Brisbane, Australia.

\section{Sample}

A convenience sample made up of parents of a child with a non-urgent illness or injury attending the study hospital's PED was used. For the purposes of this study, "non-urgent" is defined as Category 4 and 5 as per the Australasian Triage System (ATS). ${ }^{1}$ Participant recruitment took place covering a range of days and time periods, to capture the varied patient populations and conditions presenting to PED.

\section{Survey instrument}

A structured face-to-face interview was based on a modified version of a Canadian survey tool developed and used by Truman and Reutter, and further developed using the emergent themes from Woolfenden and colleagues' Australian study. ${ }^{2}$ The survey measurement tool was a 43-item questionnaire that contained both fixed-response and open-ended questions about the nature, seriousness and duration of the child's symptoms; 
the parent's at-home treatment of these symptoms; and their attempts to obtain and use professional advice before coming to the PED. The chief investigator recruited and administered the questionnaire that had been piloted in the PED among a sample of 35 parents. The pilot results guided expert review of the survey tool and clarified the conditions required for data collection.

\section{Statistics}

SPSS Version 12 was used for statistical calculations. ${ }^{4}$ Summary statistics were used to describe the demographics. Descriptive statistics, mode and frequency distribution were calculated on variables identified in the survey. The mean values of continuous variables were determined and then categorised to facilitate analysis. Categorical variables were reported in a form consistent with the literature for any associations between data or subgroups. Tests for comparisons of proportions were conducted using cross-tabulation, Pearson chi and Fisher exact test.

\section{Ethical considerations}

Ethical approval was granted from the Hospital and the University of Queensland Ethics committees. Ethical guidelines were complied with in this study.

\section{RESULTS}

\section{Child, family and socio-economic characteristics}

Out of a total of 364 parents who were approached to participate, nine refused. The final sample of 355 parents/carers were surveyed during a 3-month period in 2005. This represented $8 \%$ of the 4355 parents/carers who presented to the PED for non-urgent (ATS Category 4 and 5) care of their child. The demographic variables of the presenting child's family

Table 1 Demographic variables of sample

\begin{tabular}{|c|c|c|}
\hline Survey respondents & n (\%) & \\
\hline Carer age (years) & $\begin{array}{l}\text { Primary female care giver } \\
\mathrm{n}=349^{*}\end{array}$ & Partner $n=299 \dagger$ \\
\hline Under 20 & $2(0.6 \%)$ & 0 \\
\hline $20-29$ & $80(22.9 \%)$ & $45(15.1 \%)$ \\
\hline $30-39$ & $177(50.7 \%)$ & $147(49.1 \%)$ \\
\hline $40+$ & $90(25.8 \%)$ & $107(35.8 \%)$ \\
\hline Carer education level & Mother: $n=347$ & Partner§ $\mathrm{n}=297$ \\
\hline Year 9 or less & $15(4.3 \%)$ & $18(6.1 \%)$ \\
\hline Year 10-11 & $93(26.8 \%)$ & $71(23.9 \%)$ \\
\hline Completed year 12 & $76(21.9 \%)$ & $56(18.8 \%)$ \\
\hline Postsecondary education & $163(47.0 \%)$ & $152(51.2 \%)$ \\
\hline Health insurance status & $\mathrm{n}=355$ & $(\%)$ \\
\hline $\begin{array}{l}\text { No health insurance (Medicare } \\
\text { only) }\end{array}$ & 218 & $(61.4 \%)$ \\
\hline Private health insurance & 137 & $(38.6 \%)$ \\
\hline SEIFA & $\mathrm{n}=335^{* *}$ & $(\%)$ \\
\hline Most disadvantaged & 36 & $(10.7 \%)$ \\
\hline Disadvantaged & 61 & $(18.2 \%)$ \\
\hline Least disadvantaged & 238 & $(70.1 \%)$ \\
\hline Best description of family & $\mathrm{n}=355$ & $(\%)$ \\
\hline Living with two natural parents & 255 & $(71.2 \%)$ \\
\hline Mother alone & 47 & $(14.0 \%)$ \\
\hline $\begin{array}{l}\text { All other family types (including } \\
\text { living with grandparents) }\end{array}$ & 53 & $(14.8 \%)$ \\
\hline
\end{tabular}

*Sample: $n=349$.
+Sample: $n=299$.
\$Missing data: $n=347$.
§Missing data: $n=297$.
TSocio-Economic Indices For Areas; 2001 census of population. Australian Bureau of
Statistics.
**Missing data: $n=335$.

as indicated by the survey respondent are listed in table 1. Female carers (mother, grandmother, step or foster mothers) made up the majority of those who were accompanying a child to PED (308: $87 \%$ ) with male carers (father, grandfather, stepfather) accounting for $13 \%$ (47). The majority of female carers were aged between $30-39$ years $(51 \%)$. The remainder were evenly divided between the 20-29 years and 40+ year's age groups. Nearly half of the mothers had completed postsecondary education (47\%). Mother's partners were in a similar age group (49\%: 30-39 years) and (51\%) had completed postsecondary education.

The majority of the children surveyed did not have private health insurance (61\%) but lived in the least disadvantaged areas $(70 \%)$. Most children (71\%) resided with two natural parents. The children presenting to PED were most likely to come from two child families (44\%), and the presenting child was most likely to be the youngest sibling across all family size groupings. Just over half of the children were male (52\%) with an overall mean age of 5.5 (SD 4.4) years (12 months or less, $17 \%$; $13-59$ months, 38\%; $60+$ months, $45 \%)$. The characteristics of the families surveyed are listed in table 1.

\section{Reason for attendance}

The majority of children who attended the PED with a nonurgent condition were brought in for treatment of an illness. A wide range of illnesses presented, which have been categorised by the first description used by the carer. Children who presented with fever often had other symptoms, which usually included features of respiratory and gastrointestinal illness and lethargy, headaches and rashes (see table 2).

Injuries occurred most commonly in the home environment (45\%), with a third of these injuries resulting from falls. Nearly a third of sport injuries were from football (all codes). Just over half $(54 \%)$ of the parents/carers had noticed the illness/injury within the preceding $24 \mathrm{~h}$ with $27 \%$ attending the PED within $4 \mathrm{~h}$ of the onset of illness/injury.

A total of $60 \%$ of parents ranked perceived severity of their child's illness as the primary or secondary reason for PED attendance, with expertise of doctors (24\%) as the next highest ranked reason for attendance. Fourteen per cent of parents primarily ranked their belief that the child would end up in hospital anyway as a factor of influence on their decision to come to the PED. Another 18\% felt that the hospital was a "one stop shop" where they knew that medical, radiology, pathology and pharmacy were all available in one location.

\section{Perceived seriousness}

The perceived seriousness was not associated with whether the presentation to the PED was for an injury or illness $(p=0.22)$. The

Table 2 Clinical characteristics

\begin{tabular}{lc}
\hline Injury & $\mathrm{n}=137^{*}$ \\
Home-related & $62(45.3 \%)$ \\
School & $8(5.8 \%)$ \\
Sport & $35(25.5 \%)$ \\
Other (playground, motor car accidents, falls) & $32(23.4 \%)$ \\
IIIness & $\mathrm{n}=216^{*}$ \\
Fever & $44(20.4 \%)$ \\
Gastrointestinal & $28(13.0 \%)$ \\
Respiratory tract & $54(25.0 \%)$ \\
Other (including preparation for diagnostic procedures) & $61(28.2 \%)$ \\
Infection & $23(10.6 \%)$ \\
Postoperative complication & $6(2.8 \%)$ \\
\hline *Incomplete data: $\mathrm{n}=353$ &
\end{tabular}


severity of the injury or illness as perceived by the parents was cited as a major reason for presenting to the PED with their child. The majority of parents/carers (57\%) rated their child's condition as moderately serious with only $11 \%$ regarding their child's condition as very serious; $15 \%$ not serious and $17 \%$ not sure.

A potential indicator of parental perception of seriousness was the treatment provided before deciding to come to the PED. Use of medication and first aid were commonly identified. Paracetamol and Ibuprofen were the most commonly used medications for injury and illness. Forty-five per cent of parents/carers administered some form of medication for injury or illness, prior to presenting to the PED. With increasing maternal age, it was less likely that a child would receive medication before attending at the PED and less likely to call the GP for advice before presentation at the PED.

Parents with children under 12 months of age were just as likely to believe that their child's illness or injury was moderate to very serious in nature $(p=0.63)$ as parents with older children $(73 \%$ vs $67-68 \%$ ). Age was related to children presenting for treatment of an injury who tended to be older than those presenting for illness with $63 \%$ vs $33 \%(p<0.001)$ being 5 years or older. Children were shown to have an increasing risk of presenting with an injury with increasing age, which was inverse to illness.

Cross-tabulation of presentation time and the "nature" of the condition (injury/illness) demonstrated that parents were more likely to attend promptly if it was an injury, 54\% of injured children attended within $4 \mathrm{~h}$ of the injury occurring, whereas $9 \%$ of children who were ill attended within $4 \mathrm{~h}$ of presentation of symptoms of illness being noticed (see table 3 ).

\section{Service provision}

Ninety-five per cent of children had their own GP (primary care provider), and $16 \%$ had a paediatrician. Small percentages of children attended Outpatients Department at the hospital $(11 \%)$ or considered that they regularly attended PED (4\%).

\section{Previous medical care}

One-third of children reported a similar injury or illness in the past. A third of the children attending the PED (34\%) had visited a GP three to five times in the preceding 12 months, with $9 \%$ of children visiting a GP more than 10 times. The advice sought before attending the PED for this injury or illness included contacting the local GP $(35 \%)$ or paediatrician $(4 \%)$ or telephoning the PED (11\%). However, 35\% had not sought any advice prior to attending the PED. Of the 232 parents who did seek some advice, almost half (48\%) were advised to take their child to the PED. Seventy-seven per cent of parents considered taking their child to the GP for this injury or illness. (see fig 1).

Carers reported that since the child had become unwell, $39 \%$ of the study population had received treatment at another healthcare facility. Sixty-one per cent of children who attended a healthcare facility prior to the PED were directed and/or referred to attend the PED for further advice and treatment (see table 4).

Table 3 Illness and injury and when care was sought

\begin{tabular}{lrrr}
\hline $\begin{array}{l}\text { When injury or illness } \\
\text { noticed }\end{array}$ & Injury & Illness & p Value \\
\hline Within $4 \mathrm{~h}$ & $77(54.2 \%)$ & $20(9.4 \%)$ & \\
Within $4-24 \mathrm{~h}$ & $35(24.6 \%)$ & $61(28.6 \%)$ & \\
$2-7$ days & $21(14.8 \%)$ & $88(41.3 \%)$ & \\
$>7$ days & $9(6.3 \%)$ & $44(20.7 \%)$ & \\
Total & $142(100 \%)$ & $213(100 \%)$ & $<0.001$ \\
\hline
\end{tabular}

When parents were asked if they had contacted the PED before visiting (as opposed to requesting advice as above) 20\% had contacted PED, to enquire either as to whether they should attend $(19 \%)$ or as to the length of the waiting time. Sixteen per cent of children attended with an injury or illness that related to a condition that the child was already known to have, and of these, $16 \%$ of children suffered chronic illness.

\section{Cost and health insurance}

Australia's universal healthcare system "Medicare" provides free care at public hospital emergency departments and subsidises (or fully reimburses), GP fees. Parents were asked if "out of pocket expense" was a factor influencing the decision to come to the PED. Two per cent rated this as a primary factor, and $4 \%$ as a secondary factor out of eight choices $(n=353)$. Most carers ranked expense in seventh position (35\%).

\section{Hospital admission}

During the duration of data collection, 11\% of all Triage 4 and $15 \%$ of all Triage 5 Categories that presented to the PED were admitted. The admission rate of the study group was $11 \%$.

\section{DISCUSSION \\ Perceived seriousness}

This study found that parents presented with their unwell or injured child at the PED due to the parent's perception of the seriousness of the illness or injury. The factors that contributed to the perceived seriousness were influenced by whether it was an injury or illness and the duration of symptoms. Results from previous studies identified perceived seriousness and parental triage as major reasons for presentation at the PED. ${ }^{235}$ Other studies also found that the concern about the severity of the child's illness or injury was the most frequent reason for visiting an emergency facility. ${ }^{67}$ From this perspective, it can be argued that addressing the entire issue of attendance at a PED in terms of appropriateness is incorrect, as parents engage in "appropriate" behaviour before presentation. Presentation occurs from the parent's perception of the need to seek care for their child.

Care-giving measures are a potential indicator of parental perceived seriousness. The most common measure used, as identified in this study, was that of administration of symptom relieving medication. Truman and Reutter's finding that $45 \%$ of parents administered medication prior to seeking attention is a similar finding to this study in the instance of injury, but this was surpassed by this study's finding that $98 \%$ of children with illness received medication before presentation to the $\mathrm{PED}{ }^{2}$ This would seem to reflect that parents will try to provide treatment for their child. Medicating the child also reflects that parental triage has occurred before presentation at the PED. Previous attendance at a GP for this illness (without resolution) or referral from this primary care provider potentially provides another source of medication before presentation (eg, antibiotic use).

\section{Attendance factors}

\section{Previous medical care}

The results of this study have shown that children were more likely to have accessed a GP in the preceding 12 months than they did the emergency department. Although the care givers were not asked if they had ever previously attended this emergency department, some comparison can be made in this study where $49 \%$ of those presenting had not presented in the previous 12 months. This is the same finding as that described by Hendry and colleagues. ${ }^{8}$ Consistent with these findings, the perception that many children use the emergency department as their sole source of primary care is unsupported. 


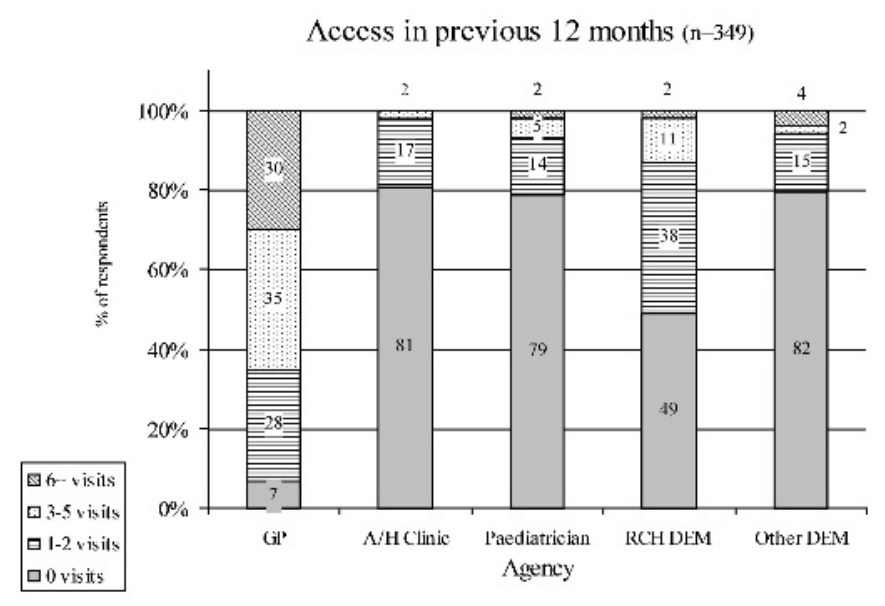

Figure 1 Number of visits to health providers in previous 12 months. Data were missing for six respondents. $n=349$. A/H Clinic, after-hours medical centre; DEM, Department of Emergency Medicine; GP, general practitioner-primary care provider; RCH, Royal Children's Hospital.

\section{Advice sought}

Alternative care to that of the PED was considered by the majority of parents of children with a non-urgent illness or injury. Of the population that attended another healthcare facility (GP/paediatrician, A/H medical centre, other hospital ED's, pharmacist) prior to attendance at this emergency department $n=139$ (39\%), the majority (61\%) were directed and or referred to the PED for further advice and treatment. This is an area that is often overlooked in discussion on presentation patterns, but it is an important factor. Nearly a quarter of parents presenting at the PED with their children for non-urgent care were there because they could not visit a GP. A third of presentations to the PED were attributable either to a lack of access to GPs or because GPs do not have the facilities to care for children that then proceed to present at the PED for non-urgent care. Eighteen per cent of the study population attended the PED, as they were unhappy with the first facility or wanted a second opinion.

Children with chronic illness are another area that appears under-researched. Often when the child's condition becomes acute (eg, cystic fibrosis), the child will require specialised care and, although not triaged as a high acuity, will be beyond the scope of the GP in the management of the condition, and invariably these acute incidents occur "out of hours."

\section{Limitations and strengths}

The sample size for this study represented $10 \%$ of the previous years attendance for ATS 4 and 5. A high response rate was obtained (97.5\%) primarily due to researcher administration. The scope of the study was limited by the time and funding available. Representativeness was tested through comparison of the whole population of ATS 4 and 5 presentations during this time with those surveyed. This demonstrated that sampling times and frameworks did not compromise the sample against either the PED or the catchment area population. Recognised diagnostic groupings were not used for two reasons. First, large numbers would have been required to obtain statistical significance, and second, they did not reflect the purpose of the research to identify causality and reason for attendance at the PED.

Because of the nature of the PED, no delineation could be made as to the type of presentation patterns at any time or any day of the week within the study data.
Table 4 Reason for coming to the paediatric emergency department following attendance at another health facility for this injury/illness

\begin{tabular}{lc}
\hline Directed by other facility & $46(31.5 \%)$ \\
Referred & $43(29.5 \%)$ \\
Unhappy & $7(4.8 \%)$ \\
Second opinion & $19(13.0 \%)$ \\
Other reason & $31(21.2 \%)$ \\
Total & $146(100 \%)$ \\
\hline
\end{tabular}

Researcher administration of the survey helped to ensure the validity and reliability of the responses collected.

\section{CONCLUSION}

This study of why parents present to the emergency department for non-urgent care has identified major factors that influence this choice. This study is the first to compare inclusive population data with paediatric emergency department data that has shown that socio-economic factors are not significant unless taken in the context of the whole population.

An important finding of this study has been that more than two-thirds of parents considered or took their child to a GP before presentation at the paediatric emergency department and that a quarter of presentations were unable to access a GP. This study highlights the deficiencies in the current primary care service available to families and the perception that not all cases deemed as non-urgent by the emergency department are able to be dealt with in a GP practice. Third, but of no less importance, is the factor of parental triage, that parents do assess their child's health before presenting at the paediatric emergency department.

In recognising that presentation is multifactorial, an issue that needs to be addressed is that there will always be nonurgent presentations at the paediatric emergency department, and service delivery and funding models need further development to address the increasing paediatric requirements for care. Funding needs to be directed to provide this from a macro level (government) and at a micro level (within the hospital and emergency department). The previous model of appropriateness of presentation needs to be discarded.

Funding: The chief investigator was awarded a scholarship to undertake this study by the Royal Children's Hospital Foundation, Brisbane.

Competing interests: None.

Ethics approval: Ethics approval was provided by the Royal Children's Hospital, Ethics Committee and University of Queensland Ethics Committee.

Provenance and peer review: Not commissioned; externally peer reviewed.

\section{REFERENCES}

1. Australian College of Emergency Medicine. The Australian Triage Scale. Carlton: Australian College of Emergency Medicine, 2000

2. Truman CD, Reutter L. Care-giving and care-seeking behaviours of parents who take their children to an emergency department for non-urgent care. Can J Public Health 2002;93:41-6.

3. Woolfenden S, Ritchie J, Hanson R, et al. Parental use of a paediatric emergency department as an ambulatory care service. Aust N Z J Public Health 2000;24:204-6.

4. SPSS. SPSS version 12 base user guide. Chicago: SPSS, 2003.

5. Smith RD, McNamara JJ. Why not your pediatrician's office? A study of weekday pediatric emergency department use for minor illness care in a community hospital. Pediatr Emerg Care 1988;4:107-11.

6. Al-Hay A, Boresli M, Shaltout AA. The utilisation of a paediatric emergency room in a general hospital in Kuwait. An Trop Paediatr 1997;7:387-95.

7. Oberlander TF, Pless IB, Dougherty GE. Advice seeking and appropriate use of pediatric emergency department. Am J Dis Child 1993;147:863-7.

8. Hendry SJ, Beattie TF, Heaney LK. Minor illness and injury: Factors influencing attendance at a paediatric accident and emergency department. Arch Dis Child 2005;90:629-33 